\title{
CLIMA ORGANIZACIONAL EM UMA UNIDADE DE ALIMENTAÇÃO E NUTRIÇÃO
}

\author{
ORGANIZATIONAL CLIMATE IN A FOOD AND NUTRITION UNIT
}

\author{
Carolina Maria Carneiro Scrideli \\ Universidade de São Paulo, SP, Brasil \\ E-mail:carol_usp@outlook.com \\ Julieta Mieko Ueta \\ Universidade de São Paulo, SP, Brasil \\ E-mail: julietapurame@gmail.com
}

Recebido em: 04.08.2020 - Aceito em: 10.11.2020

DOI: http://dx.doi.org/10.5902/2526629249208

RESUMO: Unidades de Alimentação e Nutrição (UAN) atendem instituições como as das áreas de saúde e de educação. Nesses locais, a gestão dos recursos humanos é essencial na manutenção de um bom Clima Organizacional (CO), ou seja, a "qualidade" do ambiente organizacional, que, conforme seus integrantes, afeta seus comportamentos. A pesquisa quantitativa e participativa em uma UAN de um campus universitário, com participação de 34 funcionários, objetivou analisar o seu grau de satisfação com a aplicação de questionário de $\mathrm{CO}$, adaptado e validado. Fatores internos (condições do local, execução da função, relações interpessoais, valorização e remuneração) e externos (necessidades básicas, condições de saúde e financeira, família e amigos, lazer) foram mensurados, mostrando que fatores externos não apresentaram aspectos negativos, mas os internos, como as condições físicas do local de trabalho, unanimemente o ruído, foram os que mais, negativamente, afetaram os índices de satisfação. Com essas constatações e diagnóstico, deve-se implementar políticas internas na instituição com a participação de uma equipe de trabalhadores para o planejamento e para a execução de melhorias.

Palavras-chave: Serviços de Alimentação; Recursos Humanos.

ABSTRACT: Food and Nutrition Units (FNU) serve institutions such as health and education. In these places, the management of human resources is essential in maintaining a good Organizational Climate (OC), that is, the "quality" of the organizational environment, according to its members, affects behavior. The quantitative and participatory research in a university campus UAN with the par- 
ticipation of 34 employees aimed to analyze their degree of satisfaction with the application of a CO questionnaire adapted and validated. Internal factors (location conditions, job performance, interpersonal relationships, valuation and remuneration) and external factors (basic needs, health and financial conditions, family and friends, leisure) were measured, showing that external factors did not present negative aspects, but internal conditions such as physical conditions, unanimously noise, were the ones that most negatively affected satisfaction. With these findings and diagnosis, internal policies must be implemented in the institution, with the participation of a team of workers to plan and execute improvements.

Keywords: Food services; Human Resources.

\section{INTRODUÇÃO}

Com o estilo de vida atual, uma parcela substancial da população recorre a alimentação fora do lar e, com isso, a demanda pelos diversos tipos de serviços de alimentação tem aumentado (Leal, 2010). Existem diversos tipos de serviços de alimentação: restaurantes de creches, de hospitais, de escolas, de hotéis, de comerciais e até vendedores ambulantes de refeições.

Este trabalho foi realizado em um tipo de serviço de alimentação denominado Unidade de Alimentação e Nutrição (UAN), que pode ser definida como uma unidade gerencial, na qual são desenvolvidas todas as atividades técnico-administrativas necessárias para a produção de refeições até a sua distribuição para coletividades sadias e enfermas, tendo como objetivo contribuir para manter, meIhorar ou recuperar a saúde do público atendido (CFN Resolução 600, 2018).

Numa UAN, a maioria da equipe é composta por profissionais técnicos que permanecem longos períodos em pé, em ambiente quente e ruidoso, lidando com pressão temporal para o preparo das refeições, com riscos de queimaduras, cortes, escorregões, muitas vezes carregando materiais pesados, entre outras condições (ABERC, 2003; Matos \& Proença, 2009; Casarotto \& Medes, 2005). Sendo assim, a própria natureza do trabalho em uma UAN pode oferecer riscos à saúde do trabaIhador. Por isso, diversas legislações sanitárias e trabalhistas, assim como normas regulamentadoras, foram necessárias para nortearem as condições estruturais e operacionais de trabalho nestes locais (Portaria CVS 5, 2013; RDC 216, 2004; NR 15).

Um ambiente de trabalho compõe-se de uma série de fatores físicos ou abstratos que se relacionam e que atuam de forma direta ou indireta na qualidade de vida das pessoas e nos resultados dos trabalhos por elas desenvolvidos (Wada, 1990). 


\section{JUSTIFICATIVA}

Dadas as características do ambiente de trabalho e a importância das UANs para a saúde da coletividade por elas atendida, é de suma importância olhar para a satisfação dos trabalhadores com relação ao seu ambiente de trabalho, identificando os pontos fortes e os pontos fracos a serem melhorados nesse ambiente. Nesse sentido, a pesquisa de Clima Organizacional (CO) pode contribuir nessa identificação e caracterização dos aspectos que interferem impactam na satisfação dos trabalhadores.

O CO pode ser definido como a "qualidade" do ambiente organizacional de acordo com a percepção de seus integrantes, influenciando em seus comportamentos (Souza, 1982; Ferreira, 2013; Maximiano, 2000). Pode ser considerado também como o resultado do estado de espírito ou de ânimo das pessoas, predominante em um ambiente de trabalho em um determinado período (Ferreira, 2013; Luz, 1995). A forma como a atmosfera e o ambiente de trabalho são percebidos e como estes implicam na satisfação com o trabalho e com a organização, no desempenho, nos padrões de interação em grupos e nos comportamentos de afastamento (como absenteísmo e rotatividade) pode ser definida como CO (Bowditch \& Buono, 2002). Nesse sentido, apesar de o CO ser algo abstrato, ele se "materializa" por meio de indicadores (absenteísmo, depredação do patrimônio, greves, conflitos interpessoais, desperdício de materiais, queixas nos serviços médicos, dentre outros) que fornecem sinais sobre sua qualidade, indicando se o clima está bom ou não (Ferreira, 2013). Um bom CO é essencial para se alcançar metas gerenciais, pois repercute no processo de trabalho e na motivação dos trabalhadores (Peruzzo et al, 2019).

Estudos do CO servem como base para ações corretivas ou de ajuste e para programas voltados para a implementação de melhorias, uma vez que auxiliam na identificação da situação atual, dos problemas e de suas causas nas organizações (Ferreira, 2013; Bispo, 2006). Além de, também, avaliar o grau de satisfação do funcionário em relação a empresa (Luz, 1995).

Pode-se considerar trabalhos como esse interessantes, devido a sua abordagem específica em um restaurante, uma vez que se verifica escassez de pesquisas neste setor (Maranhão \& Sá, 2019). Esses autores consideram que trabaIhadores que possuam um emprego em um ambiente de trabalho atraente e que atendam às suas expectativas poderão responder de forma favorável as demandas organizacionais. No entanto, para atender a essas necessidades, deve-se encontrar mecanismos para reconhecê-las. Justifica-se, portanto, a importância da execução de pesquisas nesse sentido (Berry \& Parasuraman, 1995; Lovelock \& 
Wright, 2006; Maranhão \& Sá, 2019) pelo pouco que se sabe, ou se presume por suposições equivocadas, a respeito do que desejam e sentem os empregados.

\section{OBJETIVO}

O objetivo deste estudo foi avaliar o CO da Seção de Alimentação (SCALIM) da Prefeitura do Campus da Universidade de São Paulo de Ribeirão Preto (PUS$\mathrm{P}-\mathrm{RP}$ ) através da percepção de seus servidores, de modo que essa avaliação possa ser um instrumento norteador para a elaboração de propostas relacionadas à promoção da qualidade do ambiente de trabalho, visando o bem-estar nesse local e a adoção de políticas internas na instituição. Para isso, foram coletadas informações através de variáveis relacionadas aos dados pessoais e profissionais dos integrantes e aos fatores externos e internos ao ambiente de trabalho que compõe o CO da SCALIM da PUSP - RP.

\section{MÉTODOS}

A Pesquisa foi realizada na SCALIM do Campus da USP de Ribeirão Preto que serve diariamente cerca de 300 cafés da manhã, 2500 almoços e 1200 jantares para alunos de 8 unidades de ensino superior e visitantes autorizados.

Participaram deste estudo 34 servidores públicos estaduais contratados pela USP, sendo: nutricionistas, técnicos em nutrição, auxiliares administrativos, cozinheiros, auxiliares de cozinha, dentre outros auxiliares. Excluíram-se estagiários e funcionários de empresas terceirizadas que prestam serviços na seção.

Para a coleta de dados, utilizou-se um questionário para a avaliação do $\mathrm{CO}$, validado e baseado em um novo modelo de pesquisa de Clima Organizacional proposto por Bispo (2006). O autor reuniu alguns dos principais modelos existentes de pesquisa de Clima Organizacional, considerados clássicos, e a partir deles criou o seu próprio modelo, enfatizando que não se tratava de um modelo fechado, que, portanto, não foi patenteado, podendo, então, sofrer modificações e ser adaptado a diferentes tipos de instituições. No questionário, empregaram-se afirmações contendo cinco alternativas de respostas utilizando a escala de Likert (1932) para medir os níveis de concordância ou discordância a estas afirmações. Para algumas questões, essas cinco alternativas de respostas não se aplicavam: dados profissionais e pessoais. Tal questionário foi composto por questões e afirmações relacionadas: aos dados pessoais (5); aos dados profissionais (4); aos fatores externos à seção (6) e aos fatores internos a seção (16). 
Os referidos questionários foram impressos e entregues aos respondentes que os preencheram, sem a necessidade de identificação. Cada participante selecionou o melhor horário para o preenchimento, sendo que o tempo disponível variou entre quinze a trinta minutos. Em seguida, o questionário foi depositado em uma caixa/urna, para posterior análise. Previamente, a pesquisa foi aplicada a uma amostra piloto.

Os dados coletados foram inseridos em planilhas da Microsoft Office Excel $2013^{\circledR}$ para tabulação e foram analisados de acordo com a escala de Likert (1932), contendo cinco alternativas de respostas: discordo totalmente, discordo, não concordo nem discordo, concordo e concordo totalmente.

As concordâncias demonstravam satisfação, sendo favorável ao bom $\mathrm{CO}$, e as discordâncias assinaladas demonstravam insatisfação, sendo desfavorável ao CO. A opção pela alternativa "não concordo nem discordo" na escala pode ser considerada neutralidade, assim como indicar insegurança em manifestar opinião e/ou indiferença em relação ao ambiente de trabalho, ou até mesmo em casos nos quais a pergunta não se aplicava.

Este trabalho foi submetido ao Comitê de Ética em Pesquisas do Hospital das Clínicas da Faculdade de Medicina de Ribeirão Preto - USP e aprovado conforme CAAE de número: 48078414.9.0000.5440.

\section{RESULTADOS E DISCUSSÃO}

\subsection{Dados sócio demográficos}

A SCALIM era composta por uma equipe de 39 servidores públicos estaduais contratados pela USP em regime CLT predominantemente feminina (68\%), com $62 \%$ possuindo idade acima dos 49 anos e, dentre estes, 82,35\% trabalhavam na seção há mais de cinco anos. Quanto à escolaridade, $88 \%$ possuíam nível adequado para exercer a função. Para o cargo de auxiliar de cozinha, a USP exigia no mínimo ensino fundamental completo, enquanto para o de cozinheiro exigia ensino fundamental completo e mais um ano de experiência relacionada às atividades a serem desempenhadas ou de curso profissionalizante na área de atuação. Justificando o nível de escolaridade da maioria dos profissionais da SCALIM, 6\% dos participantes desta pesquisa não concluíram o ensino fundamental, sendo, provavelmente, admitidos em uma época em que a Universidade adotava formas de recrutamento e de seleção diferentes das atuais.

Entre os participantes da pesquisa, $65 \%$ possuíam cônjuges e a média de filhos entres os respondentes foi de 2,05 por respondente. 


\subsection{Dados profissionais}

Dentre os servidores da SCALIM que participaram da pesquisa, $85 \%$ possuem cargos operacionais, sendo na maioria cozinheiros e auxiliares de cozinha.

Quanto ao tempo de trabalho na empresa, 29 dos 34 participantes trabaIham na instituição há mais de 5 anos, sendo 3 deles com mais de 25 anos.

Em relação ao horário de trabalho, 91\% dos servidores entrevistados trabalhavam no período matutino/vespertino, justificado pelo horário em que havia uma maior demanda de atendimento. Nesse horário, eram servidos $2 / 3$ do total de refeições do dia, também, eram realizadas as manutenções, as entregas, o recebimento e o armazenamento de mercadorias, e ainda o atendimento administrativo aos usuários da SCALIM e as reuniões com os chefes, os gestores e os setores envolvidos (divisão financeira, serviço de pessoal, etc). Além disso, neste horário concentrava-se o maior número de profissionais com restrições médicas, como lesões por esforço repetitivo e distúrbios osteomusculares. Assim, a soma de esforços de cada membro da equipe era fundamental para a superação das deficiências relacionadas aos recursos humanos e para o atendimento da demanda do restaurante universitário.

A maioria dos servidores públicos estaduais da SCALIM foram contratados através de concurso público, apenas uma minoria (18\%) foi admitida em uma época em que a forma de contratação era por indicação.

\subsection{Fatores externos à SCALIM}

Embora os fatores externos tenham origem fora do âmbito da empresa, eles exercem influência direta no comportamento, nas ações e nas decisões dos funcionários dentro do local de trabalho, por isso não devem ser negligenciados numa pesquisa de $\mathrm{CO}$. Caso a empresa não possa atuar diretamente sobre eles, pode tentar atuar sobre os seus efeitos nos funcionários. Os fatores externos ao trabalho são primordiais para a ação do indivíduo. Dessa forma, as vivências extratrabalho, a qualidade de vida, as condições de saúde, os sentimentos sobre segurança, a moradia, a alimentação, a política e a economia podem influenciar positiva ou negativamente no comportamento das pessoas também dentro das instituições (Ferraz \& Lopes, 2015).

Modernamente, a organização do trabalho vem se modificando à medida em que as transformações do mundo ganham proporções maiores e influenciam definitivamente a vida das pessoas e das organizações. Empresas alinhadas com o bem-estar dos funcionários percebem a importância de se proporcionar um am- 
biente de trabalho que atenda às necessidades físicas e mentais, promovendo a satisfação dos trabalhadores e favorecendo melhores condições de vida a essas pessoas (Maranhão \& Sá, 2019).

A Tabela 1 demostra os números absolutos (n) e os percentuais (\%) de concordâncias e discordâncias com as afirmações relacionadas aos fatores externos que compõem o CO na SCALIM.

Tabela 1 - Fatores Externos à SCALIM

\begin{tabular}{|c|c|c|c|c|c|c|}
\hline Variáveis & $\begin{array}{l}{ }^{*} \mathrm{DT} \\
\mathrm{n}(\%)\end{array}$ & $\begin{array}{l}{ }^{* * D} \\
n(\%)\end{array}$ & $\begin{array}{c}{ }^{* \star *} \mathrm{NCND} \\
\mathrm{n}(\%)\end{array}$ & $\begin{array}{l}{ }^{\star \star \star \star} \mathrm{C} \\
\mathrm{n}(\%)\end{array}$ & $\begin{array}{c}{ }^{\star \star \star \star \star} \mathrm{CT} \\
\mathrm{n}(\%)\end{array}$ & $\begin{array}{c}\star \star \star \star \star \star \star N R \\
\mathrm{n}(\%)\end{array}$ \\
\hline \multicolumn{7}{|l|}{$\begin{array}{c}\text { Necessidades } \\
\text { básicas }\end{array}$} \\
\hline Alimentação & $1(3 \%)$ & $0(0 \%)$ & $0(0 \%)$ & $10(29 \%)$ & $23(68 \%)$ & $0(0 \%)$ \\
\hline Segurança & $3(9 \%)$ & $1(3 \%)$ & $0(0 \%)$ & $8(24 \%)$ & $22(65 \%)$ & $0(0 \%)$ \\
\hline Saúde & $1(3 \%)$ & $0(0 \%)$ & $1(3 \%)$ & $9(26 \%)$ & $23(68 \%)$ & $0(0 \%)$ \\
\hline Aceitação social & $5(15 \%)$ & $2(6 \%)$ & $8(24 \%)$ & $17(50 \%)$ & $2(6 \%)$ & $0(0 \%)$ \\
\hline $\begin{array}{l}\text { Realização } \\
\text { Saúde }\end{array}$ & $0(0 \%)$ & $1(3 \%)$ & $8(24 \%)$ & $14(41 \%)$ & $10(29 \%)$ & $1(3 \%)$ \\
\hline Físico normal & $0(0 \%)$ & $5(15 \%)$ & $6(18 \%)$ & $14(41 \%)$ & $9(26 \%)$ & $0(0 \%)$ \\
\hline Mental normal & $0(0 \%)$ & $1(3 \%)$ & $1(3 \%)$ & $18(53 \%)$ & $14(41 \%)$ & $0(0 \%)$ \\
\hline Emocional normal & $0(0 \%)$ & $4(12 \%)$ & $3(9 \%)$ & $24(71 \%)$ & 3 (9\%) & $0(0 \%)$ \\
\hline \multicolumn{7}{|l|}{ Situação financeira } \\
\hline Moradia & $2(6 \%)$ & $2(6 \%)$ & $4(12 \%)$ & $9(26 \%)$ & $17(50 \%)$ & $0(0 \%)$ \\
\hline Transporte & $3(9 \%)$ & $1(3 \%)$ & $4(12 \%)$ & $12(35 \%)$ & $14(41 \%)$ & $0(0 \%)$ \\
\hline Padrão de vida & $1(3 \%)$ & $1(3 \%)$ & $7(21 \%)$ & $14(41 \%)$ & $11(32 \%)$ & $0(0 \%)$ \\
\hline Situação financeira & $3(9 \%)$ & $4(12 \%)$ & $9(26 \%)$ & $9(26 \%)$ & $9(26 \%)$ & $0(0 \%)$ \\
\hline Patrimônio & $2(6 \%)$ & $4(12 \%)$ & $6(18 \%)$ & $12(35 \%)$ & $10(29 \%)$ & $0(0 \%)$ \\
\hline Alimentação & $1(3 \%)$ & $2(6 \%)$ & $6(18 \%)$ & $12(35 \%)$ & $13(38 \%)$ & $0(0 \%)$ \\
\hline Educação & $1(3 \%)$ & $3(9 \%)$ & $7(21 \%)$ & $12(35 \%)$ & $11(32 \%)$ & $0(0 \%)$ \\
\hline \multicolumn{7}{|l|}{ Convivência familiar } \\
\hline Cônjuge & $1(3 \%)$ & $1(3 \%)$ & $4(12 \%)$ & $10(29 \%)$ & $12(35 \%)$ & $6(18 \%)$ \\
\hline Filhos & $1(3 \%)$ & $0(0 \%)$ & $0(0 \%)$ & $10(29 \%)$ & $17(50 \%)$ & $6(18 \%)$ \\
\hline Com quem moram & $1(3 \%)$ & $0(0 \%)$ & $1(3 \%)$ & $11(32 \%)$ & $19(56 \%)$ & $2(6 \%)$ \\
\hline Atenção para a família & $0(0 \%)$ & $2(6 \%)$ & $5(15 \%)$ & $11(32 \%)$ & $15(44 \%)$ & $1(3 \%)$ \\
\hline \multicolumn{7}{|l|}{ Vida social } \\
\hline Nível social & $2(6 \%)$ & $0(0 \%)$ & $3(9 \%)$ & $23(68 \%)$ & $6(18 \%)$ & $0(0 \%)$ \\
\hline Convívio social & $2(6 \%)$ & $1(3 \%)$ & $4(12 \%)$ & 20 (59\%) & $7(21 \%)$ & $0(0 \%)$ \\
\hline Nível cultural & $2(6 \%)$ & $5(15 \%)$ & $7(21 \%)$ & $16(47 \%)$ & $4(12 \%)$ & $0(0 \%)$ \\
\hline \multicolumn{7}{|l|}{ Férias e lazer } \\
\hline Últimas férias & $2(6 \%)$ & $3(9 \%)$ & $11(32 \%)$ & $7(21 \%)$ & $10(29 \%)$ & $1(3 \%)$ \\
\hline Próximas férias & $2(6 \%)$ & $0(0 \%)$ & $6(18 \%)$ & $12(35 \%)$ & $13(38 \%)$ & $1(3 \%)$ \\
\hline
\end{tabular}

${ }^{\star}$ DT: Discordo totalmente; ${ }^{\star \star}$ D: Discordo; ${ }^{\star \star \star}$ NCND: Não concordo nem discordo; ${ }^{\star \star \star \star}$ C: Concordo; ${ }^{* \star \star \star}$ CT: Concordo totalmente; ${ }^{* \star \star \star \star *}$ NR: Não responderam.

O trabalho em UAN pode ter impacto sobre a saúde do trabalhador devido à mudanças nos hábitos alimentares, que ocorrem comumente no período em que o trabalhador inicia suas atividades profissionais nesses locais, podendo contribuir 
inclusive para o agravamento da obesidade e de suas comorbidades como diabetes, hipertensão, dislipidemias, entre outras (Simon et al 2014).

De acordo com os resultados sobre os fatores extratrabalho, nas variáveis relacionadas às necessidades básicas, muitos servidores concordaram que suas maiores preocupações são com a alimentação, com a saúde e com a segurança. Um número menor de servidores demostrou se preocupar com a realização pessoal e com aceitação social. Ou seja, é positivo que os servidores priorizem suas necessidades básicas, no entanto, a maior parte dessas pessoas podem se encontrar em uma condição socioeconômica e cultural em que as maiores preocupações estejam relacionadas aos níveis mais básicos na hierarquia das necessidades humanas, como o nível das necessidades fisiológicas e o das necessidades de segurança. Isto considerando que os níveis mais elevados estejam relacionados às relações sociais, de estima e de auto realização (Maslow, 1954). Esses níveis mais elevados passam a ser priorizados pelo indivíduo quando os níveis mais básicos forem satisfeitos. Considerando a importância do suprimento dessas necessidades para a qualidade de vida (Ferreira, 2013), os fatores extratrabalho podem impactar no comportamento das pessoas em seus locais de trabalho (Ferraz \& Lopes, 2015). Desta forma, as instituições devem aprimorar continuamente as ações que contribuam para a satisfação dos vários níveis de necessidades, ou seja, preocupar-se em fornecer melhores condições socioeconômicas, culturais e de saúde para seus servidores.

Com relação às variáveis relacionadas à saúde, a maior parte dos servidores percebem-se em boas condições, no entanto, considerando a natureza das operações em uma UAN, pode ser significativo que mais de um terço dos participantes da pesquisa não tenham concordado que possuam condições físicas de saúde normais e que $21 \%$ não tenham assentido que possuam condições emocionais de saúde normais. Cabe ressaltar que a Organização Mundial de Saúde (OMS, 1986) define saúde como o completo bem-estar biológico, psicológico e social do indivíduo, e não apenas a ausência de doenças.

Os fatores que afetam ou determinam a saúde das pessoas representam os determinantes da saúde, dos quais $70 \%$ estão distantes da área da saúde. Os determinantes da saúde podem estar diretamente relacionados aos fatores físicos ou biológicos, mas os aspectos não-biológicos, relacionados às condições socioeconômicas e culturais, também afetam a qualidade da saúde (Carrapato et al, 2017).

Condições inadequadas que prejudicam a saúde podem impactar em absenteísmo. Segundo Ceribelli (2016), nas instituições públicas, o absenteísmo 
culmina na prestação de um serviço deficitário, com prejuízos à população e sobrecarga aos demais membros da equipe de trabalho presentes, com impacto desfavorável ao CO.

Nas variáveis relativas à situação financeira, as menos favoráveis, estão relacionadas a satisfação com a situação financeira e com o patrimônio. Quanto à convivência familiar, a percepção é positiva. A maior parte dos respondentes demonstrou-se satisfeita com suas vidas sociais, no entanto, considerando as insatisfações relacionadas a esta variável, é possível destacar o nível cultural como menos satisfatório.

Com relação às férias e lazer, a metade dos respondentes considerou-se satisfeitos com as últimas férias, no entanto a maioria $(73 \%)$ demonstrou boas expectativas com relação às próximas férias.

Em suma, de acordo com os resultados, a maioria dos participantes da pesquisa demostrou-se satisfeita com suas vidas extratrabalho. Podemos destacar como fatores externos favoráveis ao $\mathrm{CO}$ : o suprimento das necessidades básicas de alimentação, de saúde e de segurança; a satisfação com o estado de saúde; o relacionamento com as pessoas com quem se convive; o relacionamento com os filhos; e a satisfação com o convívio social. Dentre estes fatores externos, os menos favoráveis estavam relacionados à preocupação com a aceitação social e a satisfação com o nível cultural, com a situação financeira, com o patrimônio, com o estado físico de saúde e com as últimas férias.

\subsection{Fatores internos à SCALIM}

Os fatores internos se originam dentro da própria empresa, podendo, assim, atuar diretamente sobre esses fatores para tentar aprimorá-los, na tentativa de produzir melhorias para a empresa, para os clientes e para os funcionários (Bispo, 2006).

Os resultados relacionados aos fatores internos que compõem o $\mathrm{CO}$ na SCALIM serão apresentados em quatro partes: a. Fatores internos relacionados às condições físicas do local de trabalho; b. Fatores internos relacionados à execução da função; c. Fatores internos relacionados às relações interpessoais e d. Fatores internos relacionados às recompensas e ao desenvolvimento profissional, conforme as Tabelas 2, 3, 4 e 5.

A Tabela 2 apresenta os números absolutos (n) e os percentuais (\%) de concordâncias e discordâncias com as afirmações relacionadas aos fatores internos que compõem o $\mathrm{CO}$ na SCALIM relacionados às condições físicas do local de trabalho na seção. 
Tabela 2 - Fatores internos à SCALIM relacionados às condições físicas do local de trabalho

\begin{tabular}{ccccccc}
\hline Variáveis & $\begin{array}{c}\text { DT } \\
\mathrm{n}(\%)\end{array}$ & $\begin{array}{c}\mathrm{D} \\
\mathrm{n}(\%)\end{array}$ & $\begin{array}{c}\text { NCND } \\
\mathrm{n}(\%)\end{array}$ & $\begin{array}{c}\mathrm{C} \\
\mathrm{n}(\%)\end{array}$ & $\begin{array}{c}\mathrm{CT} \\
\mathrm{n}(\%)\end{array}$ & $\begin{array}{c}\mathrm{NR} \\
\mathrm{n}(\%)\end{array}$ \\
$\begin{array}{c}\text { Condições físicas do local } \\
\text { de trabalho }\end{array}$ & & & & & & \\
Temperatura & $4(12 \%)$ & $5(15 \%)$ & $13(38 \%)$ & $7(21 \%)$ & $3(9 \%)$ & $2(6 \%)$ \\
Espaço & $2(6 \%)$ & $6(18 \%)$ & $6(18 \%)$ & $18(53 \%)$ & $1(3 \%)$ & $1(3 \%)$ \\
Mobiliário & $4(12 \%)$ & $4(12 \%)$ & $13(38 \%)$ & $10(29 \%)$ & $2(6 \%)$ & $1(3 \%)$ \\
Higiene & $0(0 \%)$ & $4(12 \%)$ & $9(26 \%)$ & $16(47 \%)$ & $4(12 \%)$ & $1(3 \%)$ \\
Instalações sanitárias & $2(6 \%)$ & $4(12 \%)$ & $4(12 \%)$ & $19(56 \%)$ & $4(12 \%)$ & $1(3 \%)$ \\
Ruído & $8(24 \%)$ & $16(47 \%)$ & $9(26 \%)$ & $0(0 \%)$ & $0(0 \%)$ & $1(3 \%)$
\end{tabular}

${ }^{{ }^{2} \mathrm{DT}}$ : Discordo totalmente; ${ }^{* *}$ D: Discordo; ${ }^{* * *}$ NCND: Não concordo nem discordo; ${ }^{* * \star \star} \mathbf{C}$ : Concordo; ${ }^{* * * *} \mathrm{CT}$ : Concordo totalmente; ${ }^{* * * * *}$ NR: Não responderam.

De acordo com os resultados, dentre os fatores internos relacionados às condições físicas do local de trabalho, pode-se destacar como mais satisfatórios, segundo a percepção dos trabalhadores: as instalações sanitárias, as condições de higiene e o ambiente de trabalho, caracterizado como espaçoso e bem setorizado, conforme oferecido pela seção. Sobre as insatisfações relacionadas às variáveis apresentadas na Tabela 2, destaca-se o descontentamento com a condição de ruído na seção, pois nenhum participante concordou que tais condições sejam satisfatórias. Cabe ressaltar que essa foi a única unanimidade observada nos resultados desse estudo, podendo ser considerada um dos principais fatores desfavoráveis ao $\mathrm{CO}$.

O Serviço Especializado de Medicina e Segurança do Trabalho do Campus da USP de Ribeirão Preto mensura os níveis sonoros em cada setor da SCALIM, periodicamente, de acordo com as normas vigentes (NR 15). A oferta de protetores auriculares aos profissionais e a realização do exame anual de audiometria ocupacional podem ser medidas preventivas. Os ruídos na seção são provenientes principalmente: do sistema de exaustão e ventilação, dos carrinhos de transporte de materiais que passam pelos largos rejuntes dos pisos e dos equipamentos específicos para cozinhas profissionais, tais como liquidificadores, misturadores, processadores de legumes, descascadores de tubérculos e máquinas de higienização de utensílios de cozinha. Além disso, no horário de distribuição das refeições, é comum se ouvir os sons do tampar e destampar dos gastronorms (recipientes de aço inoxidável) e das vozes dos funcionários e das centenas de usuários do restaurante.

Por unanimidade, o ruído é percebido como acima do limite de tolerância pelos funcionários participantes da pesquisa. Tratando-se de estudo de $\mathrm{CO}$, considerações devem ser feitas no sentido de se adequar o nível de ruído para um considerado tolerável pela equipe, aprimorando a qualidade de vida no trabalho, um requisito da saúde ocupacional. 
Além da condição de ruído, a condição de temperatura e dos mobiliários da seção não foram percebidas como satisfatórias para a maioria dos participantes da pesquisa. Dessa forma, o desconforto auditivo, o desconforto térmico e a insatisfação com os mobiliários, que pode ter relação com a situação postural e ergonômica no trabalho, podem impactar de forma desfavorável ao clima organizacional. Além disso, devemos considerar que muitas vezes a resolução de problemas como estes tem em vista a redução de indenizações trabalhistas, enquanto a visão de melhoria e a qualidade do produto e da vida dos trabalhadores têm sido menos enfatizadas (Carvalho, 2009).

Processos de adoecimento estão ligados a fatores físicos do trabalho, além dos biológicos, sociais e psicológicos, quando estes estão em conflito com as necessidades e aspirações dos trabalhadores (Honório, 1998; Paiva, 1999; Limongi-França, 2004; Rodrigues, 2007; Maranhão \& Sá, 2019). Ademais, as pessoas passam significativa parte do tempo de vida no ambiente de trabalho, o que torna necessário a constante preocupação com a segurança, o bem-estar e o conforto dos trabalhadores, além de intervenções que visem melhorias nas condições de trabalho (Alencar et al, 2013).

A Tabela 3 demostra os números absolutos (n) e os percentuais (\%) de concordâncias e discordâncias com as afirmações relacionadas aos fatores internos que compõem o CO na SCALIM relacionados à execução da função.

Tabela 3 - Fatores internos à SCALIM relacionados à execução da função

$\begin{array}{lllllll}\text { Variáveis } & \mathrm{DT} & \mathrm{D} & \mathrm{NCND} & \mathrm{C} & \mathrm{CT} & \mathrm{NR} \\ & \mathrm{n}(\%) & \mathrm{n}(\%) & \mathrm{n}(\%) & \mathrm{n}(\%) & \mathrm{n}(\%) & \mathrm{n}(\%)\end{array}$

Autonomia

Liberdade

$2(6 \%) \quad 5(15 \%) \quad 10(29 \%) \quad 11(32 \%) \quad 6(18 \%) \quad 0(0 \%)$

Procedimento rígido

$2(6 \%) \quad 9(26 \%) \quad 9(26 \%) \quad 10(29 \%) \quad 4(12 \%) \quad 0(0 \%)$

Organização da rotina

$1(3 \%) \quad 1(3 \%)$

$3(9 \%)$

$15(44 \%) \quad 14(41 \%) \quad 0(0 \%)$

Tomada de decisão

$8(24 \%) \quad 10(29 \%)$

$10(29 \%)$

$4(12 \%) \quad 1(3 \%) \quad 1(3 \%)$

Responsabilidade

Cumprimento das

funções

$0(0 \%) \quad 0(0 \%) \quad 1(3 \%) \quad 12(35 \%) 21(62 \%) \quad 0(0 \%)$

Contribuição

$0(0 \%) \quad 0(0 \%) \quad 3(9 \%) \quad 15(44 \%) \quad 16(47 \%) \quad 0(0 \%)$

Resultados acima do

esperado

$0(0 \%) \quad 1(3 \%) \quad 4(12 \%) \quad 13(38 \%) \quad 16(47 \%) \quad 0(0 \%)$

Comprometimento

$0(0 \%) \quad 0(0 \%) \quad 1(3 \%)$

$8(24 \%) \quad 25(74 \%) \quad 0(0 \%)$ 
Realização profissional

$\begin{array}{lllllll}\text { Satisfação com o cargo } & 1(3 \%) & 5(15 \%) & 2(6 \%) & 12(35 \%) & 14(41 \%) & 0(0 \%) \\ \begin{array}{l}\text { Satisfação com o } \\ \text { trabalho que realiza }\end{array} & 4(12 \%) & 3(9 \%) & 3(9 \%) & 11(32 \%) & 13(38 \%) & 0(0 \%)\end{array}$

Quantidade e qualidade de trabalho

\begin{tabular}{|c|c|c|c|c|c|c|}
\hline Volume & $1(3 \%)$ & $0(0 \%)$ & $9(26 \%)$ & $15(44 \%)$ & $9(26 \%)$ & $0(0 \%)$ \\
\hline $\begin{array}{l}\text { Importância da } \\
\text { qualidade }\end{array}$ & $1(3 \%)$ & $0(0 \%)$ & $6(18 \%)$ & 15 (44\%) & $12(35 \%)$ & $0(0 \%)$ \\
\hline $\begin{array}{l}\text { Possibilidade de } \\
\text { melhora }\end{array}$ & $0(0 \%)$ & $2(6 \%)$ & $5(15 \%)$ & $12(35 \%)$ & $13(38 \%)$ & $2(6 \%)$ \\
\hline
\end{tabular}

Os resultados deste trabalho mostraram que os maiores níveis de concordâncias estavam associados à satisfação com variáveis relacionadas à execução da sua função (Tabela 3), ou seja, relativas ao cumprimento das responsabilidades, ao comprometimento com as atividades, à contribuição para o sucesso da instituição, à organização da rotina de trabalho e à entrega de resultados acima do esperado, o que pode ser considerado favorável ao CO na seção.

Os principais focos de insatisfação observados neste estudo não estavam relacionados à execução do trabalho. No entanto, percebeu-se muitas discordâncias sobre a participação na tomada de decisão pela seção em uma das variáveis relacionadas à autonomia.

A Tabela 4 demostra os números absolutos (n) e os percentuais (\%) de concordâncias e discordâncias com as afirmações relacionadas aos fatores internos que compõem o CO na SCALIM relacionados às relações interpessoais. 
Tabela 4 - Fatores internos à SCALIM relacionados às relações interpessoais

\begin{tabular}{ccccccc}
\hline Variáveis & $\mathrm{DT}$ & $\mathrm{D}$ & $\mathrm{NCND}$ & $\mathrm{C}$ & $\mathrm{CT}$ & $\mathrm{NR}$ \\
Comunicação & $\mathrm{n}(\%)$ & $\mathrm{n}(\%)$ & $\mathrm{n}(\%)$ & $\mathrm{n}(\%)$ & $\mathrm{n}(\%)$ & $\mathrm{n}(\%)$ \\
Direção - funcionários & $2(6 \%)$ & $5(15 \%)$ & $11(32 \%)$ & $12(35 \%)$ & $4(12 \%)$ & $0(0 \%)$ \\
Segurança dizer o que pensam & $6(18 \%)$ & $5(15 \%)$ & $14(41 \%)$ & $7(21 \%)$ & $2(6 \%)$ & $0(0 \%)$ \\
Abertura da seção & $2(6 \%)$ & $3(9 \%)$ & $15(44 \%)$ & $8(24 \%)$ & $5(15 \%)$ & $1(3 \%)$ \\
Orientações para trabalho & $0(0 \%)$ & $2(6 \%)$ & $10(29 \%)$ & $14(41 \%)$ & $7(21 \%)$ & $1(3 \%)$ \\
Relacionamentos com superiores & & & & & & \\
Respeito do chefe & $1(3 \%)$ & $1(3 \%)$ & $7(21 \%)$ & $11(32 \%)$ & $13(38 \%)$ & $1(3 \%)$ \\
Respeito pelo chefe & $1(3 \%)$ & $0(0 \%)$ & $1(3 \%)$ & $14(41 \%)$ & $17(50 \%)$ & $1(3 \%)$ \\
Receptividade as sugestões & $2(6 \%)$ & $3(9 \%)$ & $11(32 \%)$ & $11(32 \%)$ & $6(18 \%)$ & $1(3 \%)$ \\
Relacionamento interpessoal & & & & & & \\
Cooperação & $8(24 \%)$ & $4(12 \%)$ & $13(38 \%)$ & $6(18 \%)$ & $1(3 \%)$ & $2(6 \%)$ \\
Adequação & $8(24 \%)$ & $5(15 \%)$ & $14(41 \%)$ & $5(15 \%)$ & $0(0 \%)$ & $2(6 \%)$ \\
Não interferência entre funcionários & $9(26 \%)$ & $9(26 \%)$ & $8(24 \%)$ & $4(12 \%)$ & $2(6 \%)$ & $2(6 \%)$ \\
antigos e novos & & & & & & \\
$\quad$ & & & & & & \\
Trabalho em equipe & $3(9 \%)$ & $5(15 \%)$ & $15(44 \%)$ & $9(26 \%)$ & $2(6 \%)$ & $0(0 \%)$ \\
Debate de assuntos importantes & $4(12 \%)$ & $6(18 \%)$ & $8(24 \%)$ & $12(35 \%)$ & $4(12 \%)$ & $0(0 \%)$
\end{tabular}

${ }^{\star}$ DT: Discordo totalmente; ${ }^{\star \star}$ D: Discordo; ${ }^{* \star \star}$ NCND: Não concordo nem discordo; ${ }^{\star \star \star \star} \mathbf{C}$ : Concordo; ${ }^{* * \star *}$ CT: Concordo totalmente; ${ }^{* * \star * *}$ NR: Não responderam.

Dentre as variáveis apresentadas na Tabela 4, podemos observar alguns fatores favoráveis, nos quais a maioria dos respondentes concordaram que respeitavam e eram respeitados por seus superiores (relacionamento com superiores) e que recebiam orientações adequadas para o trabalho (comunicação). No entanto, a maior parte das variáveis apresentadas na Tabela 4 requer atenção, pois, de acordo com os resultados, as relações interpessoais estão entre os principais focos de insatisfação, apresentando-se desfavoráveis ao CO. Dentre eles, podemos destacar as interferências no relacionamento entre funcionários novos e antigos, o relacionamento entre os funcionários da empresa e a cooperação entre os funcionários da seção.

As afirmações com maiores percentuais (entre $41 \%$ e $44 \%$ ) de respostas "não concordo nem discordo" foram encontradas nas variáveis associadas às relações interpessoais, sendo elas: "a seção está aberta a receber e reconhecer as críticas, opiniões e contribuições de seus funcionários"; "A equipe debate assuntos importantes"; "Os funcionários sentem-se seguros ao dizerem o que pensam"; e "O relacionamento entre os funcionários da empresa é adequado".

A Tabela 5 demonstra os números absolutos (n) e os percentuais (\%) de concordâncias e discordâncias com as afirmações relacionadas aos fatores internos que compõem o CO na SCALIM relacionados às recompensas e desenvolvimento profissional. 
Tabela 5 - Fatores internos à SCALIM relacionados às recompensas e desenvolvimento profissional

\begin{tabular}{|c|c|c|c|c|c|c|}
\hline Variáveis & $\begin{array}{l}\text { DT } \\
\mathrm{n}(\%)\end{array}$ & $\begin{array}{c}\mathrm{D} \\
\mathrm{n}(\%)\end{array}$ & $\begin{array}{l}\text { NCND } \\
\mathrm{n}(\%)\end{array}$ & $\begin{array}{c}\mathrm{C} \\
\mathrm{n}(\%)\end{array}$ & $\begin{array}{l}\text { CT } \\
n(\%)\end{array}$ & $\begin{array}{l}\mathrm{NR} \\
\mathrm{n}(\%)\end{array}$ \\
\hline \multicolumn{7}{|l|}{ Remuneração } \\
\hline Satisfação com o salário & $2(6 \%)$ & $8(24 \%)$ & $5(15 \%)$ & $16(47 \%)$ & $3(9 \%)$ & $0(0 \%)$ \\
\hline Adequação ao trabalho & $6(18 \%)$ & $5(15 \%)$ & $14(41 \%)$ & $7(21 \%)$ & $2(6 \%)$ & $0(0 \%)$ \\
\hline Remuneração adequada & $4(12 \%)$ & $5(15 \%)$ & $6(18 \%)$ & $12(35 \%)$ & $7(21 \%)$ & $0(0 \%)$ \\
\hline \multicolumn{7}{|l|}{ Carreira } \\
\hline Oportunidade de crescimento na carreira & $8(24 \%)$ & $5(15 \%)$ & $9(26 \%)$ & $8(24 \%)$ & $3(9 \%)$ & $1(3 \%)$ \\
\hline Aptidão para maiores responsabilidades & $4(12 \%)$ & $2(6 \%)$ & $4(12 \%)$ & $10(29 \%)$ & $13(38 \%)$ & $1(3 \%)$ \\
\hline Interesse em outro departamento & $10(29 \%)$ & $4(12 \%)$ & $10(29 \%)$ & $6(18 \%)$ & $3(9 \%)$ & $1(3 \%)$ \\
\hline Desenvolvimento e crescimento & $7(21 \%)$ & $8(24 \%)$ & $10(29 \%)$ & $5(15 \%)$ & $3(9 \%)$ & $1(3 \%)$ \\
\hline \multicolumn{7}{|l|}{ Valorização profissional } \\
\hline Valorização & $3(9 \%)$ & $3(9 \%)$ & $13(38 \%)$ & $12(35 \%)$ & $2(6 \%)$ & $1(3 \%)$ \\
\hline Aproveitamento do potencial & $5(15 \%)$ & $5(15 \%)$ & $8(24 \%)$ & $9(26 \%)$ & $6(18 \%)$ & $1(3 \%)$ \\
\hline Reconhecimento & $8(24 \%)$ & $6(18 \%)$ & $8(24 \%)$ & $9(26 \%)$ & $2(6 \%)$ & $1(3 \%)$ \\
\hline \multicolumn{7}{|l|}{ Treinamento e desenvolvimento } \\
\hline Para execução do cargo & $2(6 \%)$ & $8(24 \%)$ & $9(26 \%)$ & $8(24 \%)$ & $6(18 \%)$ & $1(3 \%)$ \\
\hline Para desenvolvimento & $2(6 \%)$ & $8(24 \%)$ & $13(38 \%)$ & $5(15 \%)$ & $5(15 \%)$ & $1(3 \%)$ \\
\hline Capacitação para o trabalho & $2(6 \%)$ & $8(24 \%)$ & $9(26 \%)$ & $7(21 \%)$ & $7(21 \%)$ & $1(3 \%)$ \\
\hline Para aprendizado contínuo & $7(21 \%)$ & $10(29 \%)$ & $9(26 \%)$ & $3(9 \%)$ & $4(12 \%)$ & $1(3 \%)$ \\
\hline \multicolumn{7}{|l|}{ Estabilidade no emprego } \\
\hline Segurança própria com a estabilidade & $2(6 \%)$ & $5(15 \%)$ & $12(35 \%)$ & $8(24 \%)$ & $6(18 \%)$ & $1(3 \%)$ \\
\hline $\begin{array}{c}\text { Segurança dos funcionários quanto a } \\
\text { estabilidade }\end{array}$ & $3(9 \%)$ & $3(9 \%)$ & $11(32 \%)$ & $11(32 \%)$ & $5(15 \%)$ & $1(3 \%)$ \\
\hline \multicolumn{7}{|l|}{ Imagem da empresa } \\
\hline Consideração bom lugar & $0(0 \%)$ & $1(3 \%)$ & $4(12 \%)$ & $19(56 \%)$ & $10(29 \%)$ & $0(0 \%)$ \\
\hline Indicação de pessoas para a instituição & $1(3 \%)$ & $1(3 \%)$ & $5(15 \%)$ & $15(44 \%)$ & $12(35 \%)$ & $0(0 \%)$ \\
\hline Ética com funcionários, clientes e parceiros & $1(3 \%)$ & $3(9 \%)$ & $11(32 \%)$ & $15(44 \%)$ & $4(12 \%)$ & $0(0 \%)$ \\
\hline Indicação de pessoas para a seção & $3(9 \%)$ & $7(21 \%)$ & $8(24 \%)$ & $11(32 \%)$ & $5(15 \%)$ & $0(0 \%)$ \\
\hline Exemplos dos gestores & $2(6 \%)$ & $3(9 \%)$ & $12(35 \%)$ & $11(32 \%)$ & $6(18 \%)$ & $0(0 \%)$ \\
\hline
\end{tabular}

"DT: Discordo totalmente; ${ }^{* \star}$ D: Discordo; ${ }^{\star * \star}$ NCND: Não concordo nem discordo; ${ }^{\star \star \star \star}{ }^{*}$ : Concordo; ${ }^{\star \star \star \star \star C T: ~ C o n c o r d o ~ t o t a l m e n t e ; ~}{ }^{* \star \star \star *}$ NR: Não responderam.

De acordo com os resultados apresentados nesta tabela, podemos observar, através dos focos de concordâncias, que alguns fatores favoráveis ao CO estavam relacionados à imagem da instituição. No entanto, dos 34 respondentes, 27 (79\%) indicariam pessoas para trabalharem na instituição e $16(47 \%)$ indicariam pessoas para trabalharem na seção, ou seja, a satisfação com a imagem da seção pode estar aquém da satisfação com a imagem da instituição.

Com relação às insatisfações, podemos observar que a maioria dos respondentes não concordou que recebia treinamento e desenvolvimento para aprendizado contínuo (treinamento e desenvolvimento), que possuía oportunidade para o crescimento de desenvolvimento profissional (carreira), que a empresa reconhecia os bons funcionários (valorização profissional) e que possuía oportunidades 
de crescimento na carreira (carreira). Além disso, $27 \%$ dos profissionais da SCALIM participantes da pesquisa afirmaram possuir interesse em outros setores da instituição.

A satisfação com a remuneração não parece ter sido um dos principais fatores desfavoráveis. Entretanto, enquanto a maioria dos respondentes concordou que a empresa remunerava adequadamente os funcionários, ou seja, que os pagamentos eram realizados nas datas corretas e de acordo com as legislações trabalhistas, além de estar satisfeita com o salário atual, poucos funcionários consideraram que a remuneração estava adequada ao trabalho que desempenhavam.

Uma síntese dos fatores favoráveis e desfavoráveis ao CO estão apresentados na Tabela 6.

Tabela 6 - Síntese dos fatores internos favoráveis e desfavoráveis ao CO na SCALIM

$\begin{array}{ll}\begin{array}{l}\text { Fatores relaciona- Fatores internos favoráveis ao } \mathrm{CO} \\ \text { dos às }\end{array} & \text { Fatores internos desfavoráveis ao } \\ \mathrm{CO}\end{array}$

Condições físicas Instalações sanitárias, condições de Ruído (principalmente), temperatura e do local de trabalho higiene, espaço amplo e setorizado mobiliário no ambiente de trabalho

Execução da fun- Cumprimento das responsabilida- Participação na tomada de decisão ção des, comprometimento com as ativi- pela seção dades, contribuição para o sucesso da instituição, entrega de resultados acima do esperado

Relações interpes- Respeito aos chefes, gerentes ges- Interferências no relacionamento entre soais tores, respeito dos superiores e re- funcionários novos e antigos, relaciocebimento de orientações adequa- namento entre os funcionários da emdas para o trabalho presa, cooperação entre os funcionários da seção

Recompensas e Consideração da instituição como Treinamento e desenvolvimento para desenvolvimento um bom lugar para trabalhar (ima- aprendizado contínuo, oportunidade profissional gem da instituição), a empresa re- para o crescimento de desenvolvimenmunera os funcionários pontual e to profissional, reconhecimento dos adequadamente do ponto de vista bons funcionários, oportunidades de legal. crescimento na carreira, adequação da remuneração ao trabalho realizado.

A pesquisa de CO é composta por uma gama de variáveis. O objetivo deste estudo não foi o de se aprofundar na discussão de cada variável, mas sim o de identificar os fatores favoráveis e desfavoráveis ao CO na percepção dos servidores da SCALIM. Neste sentido, com os resultados deste trabalho, a seção poderá estabelecer prioridades e elaborar planos de ação para tentar melhorar os seus pontos fracos, além de manter e/ou aprimorar os seus pontos fortes. 
Por um lado, este estudo apresentou pontos positivos, como o fato de que os participantes tiveram a oportunidade de expor suas opiniões, provocando a sensação de terem sido ouvidos e respeitados, sentindo-se corresponsáveis pela instituição. Fatores estes que por si sós podem impactar na satisfação e na produção dos trabaIhadores. Além disso, tiveram a oportunidade de, através de suas opiniões, contribuírem para possíveis melhorias em seu ambiente de trabalho. Essas melhorias podem estar relacionadas à melhoria da estrutura física do local de trabalho, à distribuição das atividades entre a equipe de trabalho, ao investimento em treinamentos necessários e ao desenvolvimento de pessoas, ao desenvolvimento de trabalhos relacionados à gestão de conflitos, que podem ser vistos como fonte geradora de mudanças, crescimento e desenvolvimento (Nascimento \& El Sayed, 2002), e ao aprimoramento de lideranças. A Universidade poderá optar por planos de ações que contemplem parcerias com diversos setores, otimizando assim a utilização de seus recursos.

Por outro lado, também é importante ressaltar que a pesquisa de $\mathrm{CO}$ pode ter apresentado algumas limitações, vieses e desconfortos aos participantes, considerando-se que estes expuseram suas opiniões sobre o seu local de trabalho para um pesquisador responsável que fazia parte da equipe na qual o estudo foi aplicado. Além disso, a pesquisa de $\mathrm{CO}$ em si pode gerar expectativas relacionadas a melhorias no ambiente de trabalho, o que poderá causar frustrações e desconfianças por parte dos funcionários, caso nada seja feito a partir dos seus resultados.

Macdonald e Oakman (2015), ao tratar de desordens musculoesqueléticas relacionadas ao trabalho, exacerbadas neste ambiente de UAN, enfatizam que atualmente é recomendado uma ação mais sistêmica e holística, sem compartimentalizar as condições inadequadas ou seus impactos. Sugere-se a participação dos funcionários para contribuírem na construção de ferramentas de gerenciamento que afetem as condições do trabalho. Neste sentido, esta pesquisa procurou iniciar o processo de aprimoramento destas condições, juntamente à qualidade de vida, explorando a "qualidade" do ambiente organizacional de acordo com a percepção dos servidores públicos, influenciando em seus comportamentos (Souza, 1982; Ferreira, 2013; Maximiano 2000); o que, em síntese, se qualifica como uma pesquisa de CO.

\section{CONSIDERAÇÕES FINAIS}

A pesquisa de CO realizada na SCALIM da PUSP- RP permitiu avaliar e identificar os fatores internos e externos, positivos e negativos, que compõem o referido clima. Os dados obtidos poderão contribuir para reavaliar e/ou reestrutu- 
rar vários aspectos organizacionais, tais como: políticas e estratégias de recursos humanos; estrutura física da seção; tecnologias utilizadas; comunicação; gestão de conflitos; saúde e segurança no trabalho; dentre outros. Por meio desta ferramenta, é possível detectar melhor como está o seu $\mathrm{CO}$ e a qualidade de vida de seus funcionários, assim como verificar como está o relacionamento interpessoal no local, além de levantar os aspectos que causam satisfação e insatisfação segundo a percepção dos trabalhadores. Este trabalho apresenta uma forma de levantar não só as fortalezas, mas também as adversidades na seção, porém suas soluções irão depender dos recursos disponíveis, do estilo gerencial, da política e das estratégias de gestão para os recursos humanos da instituição.

Espera-se que este trabalho contribua para a implantação de melhorias no local, e que possa vir a ajudar empresas e instituições. Isto especialmente nas Unidades de Alimentação e Nutrição, uma vez que fazem parte de um setor em que há escassez de pesquisas a identificarem os pontos fortes e os pontos insatisfatórios que afetam o CO no ambiente de trabalho. Junto a isso, aspira-se que o estudo também contribua para a discussão e a divulgação sobre esse tema importante: O Clima Organizacional, que não deve ser negligenciado.

\section{REFERÊNCIAS}

Alencar, M. C. B., Cavalcanti, T. A., \& Montrezor, J. B. (2013). Condições de trabaIho em uma cozinha industrial e distúrbios osteomusculares de trabalhadores. Cadernos de Terapia Ocupacional da UFSCar, 21(1), 155-162.

Associação Brasileira das Empresas de Refeições Coletivas. (2003). Manual $A B E R C$ de práticas de elaboração e serviço de refeições para coletividades. ABERC.

Berry, L. L.; Parasuraman, A. (1995). Serviços de Marketing: competindo através da qualidade (3rd ed.). Tradução de Beatriz Sidou. São Paulo: Maltese.

Bispo, C. A. F. (2006). Um novo modelo de pesquisa de clima organizacional. Production, 16(2), 258-273.

Bowditch, J. L.; Buono, A. F. (2002). Elementos de Comportamento Organizacional. São Paulo: Pioneira Thomson

Brasil. (2014). Resolução RDC $n^{\circ} 52$, de 29 de setembro de 2014. Altera a resolução $R D C n^{\circ} 216$, de 15 de setembro de 2004, que dispõe sobre o regulamento técnico de boas práticas para os serviços de alimentação. Diário Oficial [da] República Federativa do Brasil. 
Carrapato, P., Correia, P., \& Garcia, B. (2017). Determinante da saúde no Brasil: a procura da equidade na saúde. Saúde e Sociedade, 26, 676-689. Recuperado de https://www.scielosp.org/article/sausoc/2017.v26n3/676-689/pt/.

Casarotto, R. A., \& Mendes, L. F. (2003). Queixas, doenças ocupacionais e acidentes de trabalho em trabalhadores de cozinhas industriais. Revista Brasileira de Saúde Ocupacional, 28 (107-108), 119-126. Recuperado de http:// www.scielo. br/scielo.php?script=sci_arttext\&pid=S0303- .

Ceribeli, H. B., Inácio, R. D. O., \& Silva, M. C. D. (2016). Uma análise do absenteísmo

no setor público brasileiro. Recuperado de https://www.repositorio.ufop.br/ handle/123456789/12847

Carvalho, F. M. de. (2009). Contribuições da ergonomia para projetos em unidades de Alimentação. XIII Encontro Latino Americano de Iniciação Cientifica e IX Encontro de Pós-Graduação-Universidade do Vale do Paraíba. Recuperado de http://www.inicepg.univap.br/cd/INIC_2009/anais/arquivos/ RE_0004_0921_01.pdf

Nutricionistas, C. F. de. (2018). Resolução CFN n 600, de 25 de fevereiro de 2018. Dispõe sobre a definição das áreas de atuação do nutricionista e suas atribuições, indica parâmetros numéricos mínimos de referência, por área de atuação, para a efetividade dos serviços prestados à sociedade e dá outras providências. Diário Oficial da União, 23, 1-55.

Ferraz, R., \& Lopes, E. (2015). Satisfação no trabalho: Comparação de duas escalas de medida por meio de equações estruturais. Revista de Gestão dos Países de Língua Portuguesa, 14(1), 37-47. Recuperado de http://www.scielo. mec.pt/scielo.php?script=sci_arttext\&pid=S1645-44642015000100005

Ferreira, P. I. (2013). Clima organizacional e qualidade de vida no trabalho. Rio de Janeiro: LTC.

Honório, L. C. (1998). Cisão e Privatização: impactos sobre a qualidade de vida no trabalho de uma empresa de telefonia celular. (Master's thesis, Universidade Federal de Minas Gerais, Belo Horizonte).

Leal, D. (2010). Crescimento da alimentação fora do domicílio. Segurança Alimentar e Nutricional, 17(1), 123-132. Recuperado de https://periodicos.sbu. unicamp.br/ojs/index.php/san/article/view/8634806/2725

Likert, R. (1932). A technique for the measurement of attitudes. Archives of psychology. Recuperado de: http://www.voteview.com/pdf/Likert_1932.pdf.

Limongi-França, A. C. (2004). Qualidade de Vida no Trabalho: conceitos e práticas nas empresas da sociedade pós-industrial. São Paulo: Atlas. 
Loverlock, C., \& Wright, L. (2006). Serviços: marketing e gestão. São Paulo: Saraiva.

Luz, R. S. (1995). Clima Organizacional. Rio de Janeiro: Quality.

Macdonald, W., \& Oakman, J. (2015). Requirements for more effective prevention of work-related musculoskeletal disorders. BMC musculoskeletal disorders, 16(1), 1-9.

Maranhão, M. L., \& de Sá, M. A. D. (2019). Cultura organizacional e práticas de qualidade de vida no trabalho: um estudo de múltiplos casos em palcos organizacionais de restaurantes do Recife-PE. Revista de Carreiras e Pessoas (ReCaPe), 9(2). ISSN-e: 2237-1427,

Maslow, A., \& Frager, R. (1954). Motivation and Personality (book). Recuperado de http://self.gutenberg.org/article/whebn0045431602/motivation\%20 and\%20personality\%20.

Matos, C. H. D., \& Proença, R. P. D. C. (2003). Condições de trabalho e estado nutricional de operadores do setor de alimentação coletiva: um estudo de caso. Revista de Nutrição, 16(4), 493-502.

Maximiano, A. C. A (2000). Introdução a Administração. São Paulo: Atlas.

Nascimento, E. M.; El Sayed, K. M. Administração de conflitos. In: Mendes, J. (2002). Gestão do capital humano. Coleção gestão empresarial. V. 5. p. 47-56.

OMS - Organização Mundial de Saúde (1986). Carta de Ottawa para a promoção da saúde: primeira conferência internacional sobre promoção da saúde. Genebra.

PAIVA, K. C. M. (1999). Qualidade de Vida no Trabalho e Stress de Profissionais docentes: uma comparação entre o público e o privado. (Master's thesis, Universidade Federal de Minas Gerais. Belo Horizonte).

Peruzzo, H. E., Silva, E. S., Batista, V. C., Haddad, M. D. C. F. L., Peres, A. M., \& Marcon, S. S. (2019). Clima organizacional e trabalho em equipe na estratégia saúde da família. Revista Brasileira de Enfermagem, 72(3), 721-727.

Portaria, C. V. S. (2013). 5, de 09 de abril de 2013. DOE de, 19(04). Recuperado de http://www.cvs.saude.sp.gov.br/up/PORTARIA\%20CVS-5_090413.pdf.

Simon, M. I. S. D. S., Garcia, C. A., Lino, N. D., Forte, G. C., Fontoura, I. D. D., \& Oliveira, A. B. A. D. (2014). Avaliação nutricional dos profissionais do serviço de nutrição e dietética de um hospital terciário de Porto Alegre. Cadernos saúde coletiva, 22(1), 69-74. 
Souza, E. L. P. D. (1982). Clima e motivação em uma empresa estatal. Revista de Administração de Empresas, 22(1), 14-18.

TRABALHISTA, G. (2017). Norma regulamentadora 15. Atividades e operações insalubres. Recuperado de http://www. guia trabalhista. com. br/legislacao/ nr/nr15. Htm.

Wada, C. C. B. B. (1990). Saúde: determinante básico do desempenho. Revista alimentação e nutrição, (56), 36-38. 